\title{
The Effect of Crosstalk on the Perceived Depth From Disparity and Monocular Occlusions
}

\author{
Inna Tsirlin, Laurie M. Wilcox, and Robert S. Allison
}

\begin{abstract}
Crosstalk in stereoscopic displays is defined as the leakage of one eye's image into the image of the other eye. All popular commercial stereoscopic systems suffer from crosstalk to some extent. Studies show that crosstalk causes distortions, reduces image quality and visual comfort, and increases perceived workload. Moreover, there is evidence that crosstalk effects depth perception from disparity. In the present paper we present two experiments. The first addresses the effect of crosstalk on the perceived magnitude of depth from disparity. The second examines the effect of crosstalk on the magnitude of depth perceived from monocular occlusions. Our data show that crosstalk has a detrimental effect on depth perceived from both cues, but it has a stronger effect on depth from monocular occlusions. Our findings taken together with previous results suggest that crosstalk, even in modest amounts, noticeably degrades the quality of stereoscopic images.
\end{abstract}

Index Terms-Crosstalk, ghosting, human factors, stereo vision, three-dimensional displays.

\section{INTRODUCTION}

$\mathbf{S}$ TEREOSCOPIC displays and stereoscopic three-dimensional (S3D) applications are becoming increasingly popular in the consumer market. Recently, major film production companies have released several movies in S3D and electronics giants such as Sony and Panasonic have introduced S3D television sets. The lasting success of this new market directly depends on the quality of stereoscopic displays and the vividness of perceived depth. While stereoscopic display technology is constantly improving there are persistent problems that affect the quality of S3D images. Stereoscopic displays rely on the capability to present independent images to the left and right eyes of the viewer. An important consequence of not meeting this requirement is crosstalk, which is defined as the leakage of one eye's image into the image of the other eye. Human observers perceive crosstalk as ghost images particularly around high contrast (e.g., white on black) image features. Ghosting has been implicated as a major factor influencing viewer satisfaction with stereoscopic content [1]-[3].

All popular commercial stereoscopic viewing systems suffer from crosstalk (see [4] for a review). In time-sequential displays the left and right eye images are presented consecutively and the

Manuscript received August 10, 2010; revised December 14, 2010; accepted January 04,2011 . Date of publication January 31,2011 ; date of current version May 25, 2011. This work was supported by NSERC Discovery Grants to R. Allison and L. Wilcox and a Canada Graduate Scholarship to I. Tsirlin.

The authors are with the Centre for Vision Research at York University, Toronto, ON M3J 1P3, Canada (e-mail: itsirlin@yorku.ca; lwilcox@yorku.ca; allison@cse.yorku.ca).

Color versions of one or more of the figures in this paper are available online at http://ieeexplore.ieee.org.

Digital Object Identifier 10.1109/TBC.2011.2105630 presentation of each image is synchronized with the closing of a shutter in front of the other eye (or with other devices such as an alternating polarizer). Crosstalk occurs in these systems, among other reasons, due to slow shuttering, shutter leakage and persistence of the image to be extinguished into the temporal display window of the other eye (i.e. phosphor persistence in a CRT or plasma display) [5]. In polarized displays, the images of the two eyes are passed through orthogonal polarizing filters and then simultaneously projected on the screen. The segregation of the images is maintained by using glasses with matching orthogonal polarization in the two eyes. In polarized displays crosstalk can occur due to finite extinction in the polarizing filters (both on the projectors and in the eyewear), screen depolarization, misalignment or leakage between micropolarizer arrays and display pixels and misalignment between the polarized filters on the projectors and in the eyewear (which can also occur with head tilt in linear polarization systems) [4]. Autostereoscopic displays do not require glasses. They achieve image segregation by using sophisticated optics or viewing barriers, which direct separate light rays into the two eyes. Most of these displays allow several views of the same scene. These systems are also prone to crosstalk around the borders of adjacent views [6], [7]. Anagylph systems are widely known to exhibit the largest amounts of crosstalk. In anaglyph displays, the left and the right images are displayed simultaneously but via different color channels. For instance, the left image might be red and the right image green or cyan. The viewer wears glasses with color filters that match the colors used in creation of the anaglyphs. Crosstalk frequently occurs in anaglyph displays due to the imperfect spectral performance of the filters and mismatch with the spectral emission of the displays [8]. More sophisticated wavelength selective techniques can reduce crosstalk and provide vibrant color but some crosstalk still occurs [9].

The amount of crosstalk in a given system depends on the various system parameters and also on the measurement methods. Unfortunately, there is no comprehensive review which compares crosstalk across different systems. However, some example data have been provided for several systems. Woods and Harris [8] and Woods and Rourke [10] conducted a detailed simulation study of crosstalk in anaglyph type systems. They found that depending on the display, the glasses and the combination of colors used, crosstalk in these systems can be as high as $96 \%$ or as low as $\sim 2 \%$. Crosstalk in a parallax barrier autostereoscopic display was estimated to be around 5\% [6]. In time-sequential displays it was reported that a system combining shutter glasses with an LCD display could produce up to $8 \%$ crosstalk [11].

More is known about the perceptual consequences of crosstalk. Ghosting from crosstalk was found to cause dis- 
tortions in natural images, where the amount of perceived distortion (ghosting, double-lines) increased with the increase in crosstalk [1]. Wilcox and Stewart [3] reported that crosstalk was the most important attribute in determining image quality for $75 \%$ of their observers. They found that as crosstalk increased quality rating decreased consistently across different image brightness conditions. Pala et al. [12] found that perceived workload increased in the presence of crosstalk in a task where observers were asked to align rods in depth. Still other studies have reported reduced visual comfort with increasing crosstalk [2], [13], [14]. Furthermore, crosstalk over 5\% was found to cause a reduction in viewing comfort, especially for images containing large disparities [2].

Several studies have assessed the effect of crosstalk on depth perception. Pala et al. [12] showed that the ability to discriminate the convexity/concavity of a 3D sphere and to align two rods in depth was hindered by the presence of ghosting. In another study, observers judged depth in natural and artificial images using a Likert scale from 3 to -3 , where 3 indicated good depth and -3 indicated reversed depth [15]. It was found that increase in crosstalk resulted in degraded depth quality. Seuntiens et al. [1] asked their observers to judge the overall depth in two natural scenes using a 5-point categorical scale. They showed that the ratings of depth in the scenes depended on the disparity but not on crosstalk. The lack of an effect of crosstalk in this experiment could be due to the assessment method used as well as to the range of crosstalk values tested. It is important to note that all of the preceding experiments either considered qualitative/categorical depth perception or the ability to discriminate very small depth intervals. However the disparities in S3D displays are typically well above perceptual threshold so it is arguable the perception of depth magnitude, space and volume that should be of principal concern.

The first goal of the present work was to evaluate the effect of crosstalk on perceived depth from disparity using a more precise and direct method. That is, we used a depth estimation task, where observers were asked to indicate the amount of perceived depth in centimeters using a scale and a sliding cursor. We systematically varied the disparity and the amount of crosstalk in the stimuli to encompass a broad range of values. The experiment was run on a zero crosstalk mirror stereoscope and images were manipulated to precisely simulate varying degrees of crosstalk.

Our second goal was to assess the effect of crosstalk on depth from monocular occlusions. Monocular zones are areas that are seen only by one eye while viewing a scene binocularly. They typically arise due to the lateral separation of our eyes and the occlusion of surfaces by nearer objects or other surfaces. This fact was noted by Leonardo da Vinci, who observed that because each eye sees slightly more of one side of a sphere, no $2 \mathrm{D}$ representation can fully recreate a $3 \mathrm{D}$ scene. In their seminal paper Nakayama and Shimojo [16] used a simple stimulus which depicted a rectangle occluding a bar, to show that when the location of the bar was consistent with an occlusion interpretation (i.e. a bar visible in the right eye was placed to the right of the rectangle) then the bar was clearly seen behind the rectangle (see Fig. 1(b)). They named this phenomenon da Vinci stereopsis. Numerous subsequent experiments have confirmed

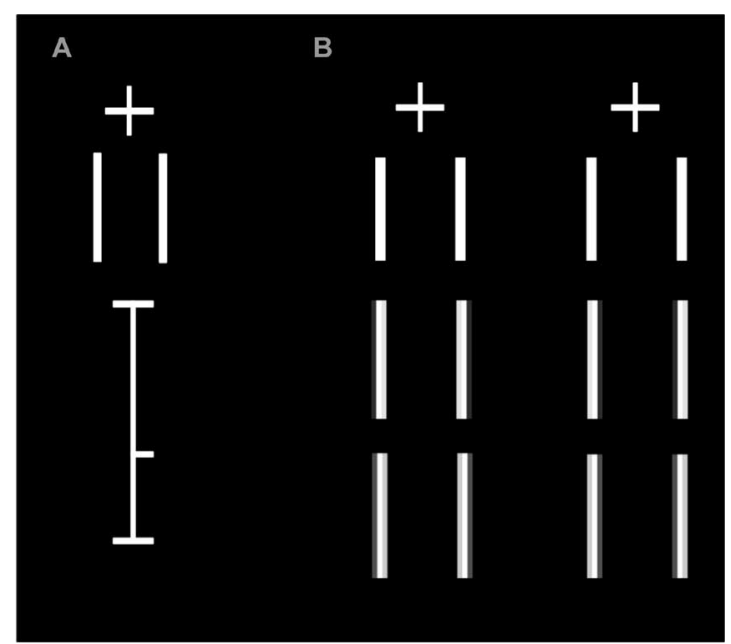

Fig. 1. Depiction of stimuli used in Experiment 1. (a) The complete display. (b) Example of stimuli arranged for free-fusion (they can be viewed with either crossed or divergent fusion). On the top row, there is no crosstalk; the middle and bottom rows have $16 \%$ and $32 \%$ crosstalk, respectively. The lines have a disparity of $10.62 \mathrm{~min}$ of arc $(1.83 \mathrm{~cm})$ with respect to the fixation.

and reinforced the finding that depth can be seen purely on the basis of monocular occlusions (for a review see [17]). It has also been reported that monocular occlusions play an important role in stereoscopic depth perception. For example, the presence of monocular occlusions can speed up depth perception [18]-[20], resolve depth order in stimuli with ambiguous disparity [21], [22], create illusory surfaces and boundaries in depth [23]-[25] and even yield quantitative depth percepts [23], [25]. Monocular occlusions also play an important role in creating quality S3D content (for a review see [26]).

Monocular occlusions are abundant in cluttered natural scenes and their importance for veridical depth perception is clear. However, to date no one has evaluated the effect of degraded monocular areas on the perception of depth in S3D displays [14]. In this paper we examine the effect of crosstalk on depth from monocular occlusions using the direct depth estimation task described above.

Results from both experiments show that crosstalk interferes with depth perception, especially in the case of monocular occlusions. For these stimuli increasing crosstalk beyond $1 \%$ causes a significant decrease in perceived depth. In the case of disparity, the effect depends on the disparity magnitude. For larger disparities crosstalk beyond $2-4 \%$ reduces perceived depth significantly. We discuss the implications of these findings for the S3D display industry and for S3D content creators.

\section{EXPERIMENT I}

In this experiment we examine the effect of crosstalk on perceived depth from binocular disparity.

\section{A. Methods}

Observers: Nine volunteers participated in the study. Two of them (IT and LW) are authors and the rest were naluml;ve as to the purpose of the study. All observers had normal or corrected-to-normal visual acuity and good stereoacuity as measured with Randot stereoacuity test (observers had to be able 
to discriminate disparity of 40 seconds of arc). The interocular distance for each observer was measured with a Richter digital pupil distance meter.

Apparatus: Scripts for stimulus presentation were executed on a G5 Power Macintosh using the Psychtoolbox package for MATLAB (v. 7.4). Stimuli were presented on a pair of CRT monitors (ViewSonic G225f) arranged in a mirror stereoscope at a viewing distance of $0.6 \mathrm{~m}$. The resolution of the monitors was set to $1280 \times 960$ pixels and the refresh rate to $75 \mathrm{~Hz}$. At this resolution and viewing distance, each pixel subtended 1.77 minutes of visual angle. The monitors were linearized using a photometer to appropriately adjust the gamma function. A chin rest stabilized head position during testing.

Stimulus: The stimulus was composed of two vertical lines $(10.6 \times 177 \mathrm{arcmin})$, one positioned $44.25 \mathrm{arcmin}$ to the left and the other 44.25 arcmin to the right of the midline of the display. The left line had an uncrossed disparity and the right line an equal crossed disparity of $3.54,7.08,10.62,14.16$, or 17.7 arcmin with respect to the plane of the display (total disparity between the lines was $7.08,14.16,21.24,28.32$ or 35.4 arcmin accordingly). To create the disparity each half-image was shifted to the left (or the right) by half the disparity. The width of the stimulus lines was chosen specifically so that at all test disparities the ghost images caused by crosstalk would not be completely spatially segregated from the stimulus line.

A short distance (53.1 arcmin) above the stimulus there was a fixation cross composed of lines with length 26.5 arcmin. The upper and lower vertical lines were presented as a Nonius line pair. In this technique one line is presented only to one eye and the other line only to the fellow eye. If the observer's eyes are correctly converged on the display these lines appear aligned; if the eyes are misconverged the misalignment will be obvious to the observer.

A vertical scale with an adjustable cursor was centered 70.8 arcmin below the stimulus. The scale was 354 arcmin in length and the cursor was 7.08 arcmin wide. Observers could move the cursor up and down the scale using a computer mouse. All the parts of the display and stimulus stereograms are shown in Fig. 1.

The screen background was set to black and the stimulus, fixation cross and the scale were light gray (grayscale 193, luminance $78.95 \mathrm{~cd} / \mathrm{m}^{2}$ ). This grayscale level was selected specifically so that for stimuli with the highest level of crosstalk the additive grayscale level will not surpass the highest possible value of 256 .

To introduce crosstalk, an attenuated version-one of $0,1,2$, $4,8,16$ or $32 \%$-of the right image was added to the left image and vice versa. The gray levels of the ghost image (where not overlapping with the real line) were $0,1.9,3.9,7.7,15.4,31.0$ and 62.0 accordingly. We ensured that our displays had enough color resolution to represent these gray levels distinctly by measuring the corresponding luminance for each gray level (10 independent measurements per gray level) using a photometer. The luminance was significantly different for all of the gray levels in both stereoscope screens (luminance 0, 0.63, 1.32, 2.70, 5.81 , 11.94, $25.54 \mathrm{~cd} / \mathrm{m}^{2}$ accordingly). Moreover, in a pilot experiment we made sure that these gray levels are also discernable perceptually by displaying the gray levels consecutively on the
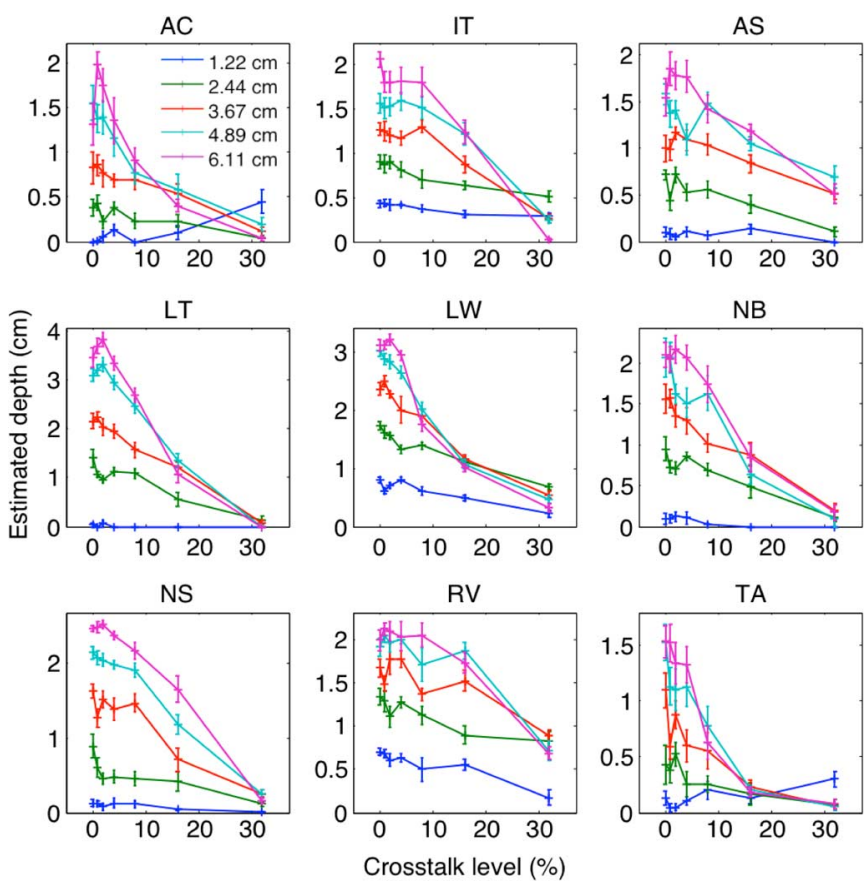

Fig. 2. Results of Experiment 1 for all observers. The abscissa shows the crosstalk levels, and the ordinate shows the depth estimates. The different colored lines represent different disparities. The depth estimates were expressed in terms of the equivalent theoretical geometric disparity that would produce the depth at the viewing distance (see text). The error bars indicate $+/-1$ standard error. Note that the ordinate does not show the same scale for all observers to account for individual differences.

screen and asking a subset of observers whether they could see a difference between consecutive gray levels. The gray levels were clearly distinguishable for the observers.

Procedure: The observers were asked to use the mouse to adjust the cursor on the scale so that the interval between the cursor and bottom of the scale matched the depth perceived between the two test lines. They were encouraged to use the fixation cross to stabilize their gaze while viewing the stimulus. Observers were free to move their eyes between the measurement scale and the stimulus and the viewing time was not restricted. The experiment consisted of two sessions where each condition (crosstalk level + disparity) was presented 10 times in random order. In total there were 35 different conditions ( 7 crosstalk levels $\times 5$ disparities) and 175 trials per session for 350 trials in total. The experiment took place in a completely dark room.

Statistical Analysis: To analyze the data we used a nonparametric Wilcoxon signed-rank test. All statistical analyses used alpha level of 5\% and a one-tailed test.

To see at which level of crosstalk the estimated depth becomes significantly reduced we compared each of the non-zero crosstalk conditions to the zero crosstalk condition using multiple paired tests. We conducted this analysis for each disparity separately. All statistical analyses were performed using the statistical software package $R$.

\section{B. Results}

Mean data are shown in Fig. 3; data for individual observers are shown in Fig. 2. Angular disparities of the stimuli were converted to theoretical depth in centimeters in the following 

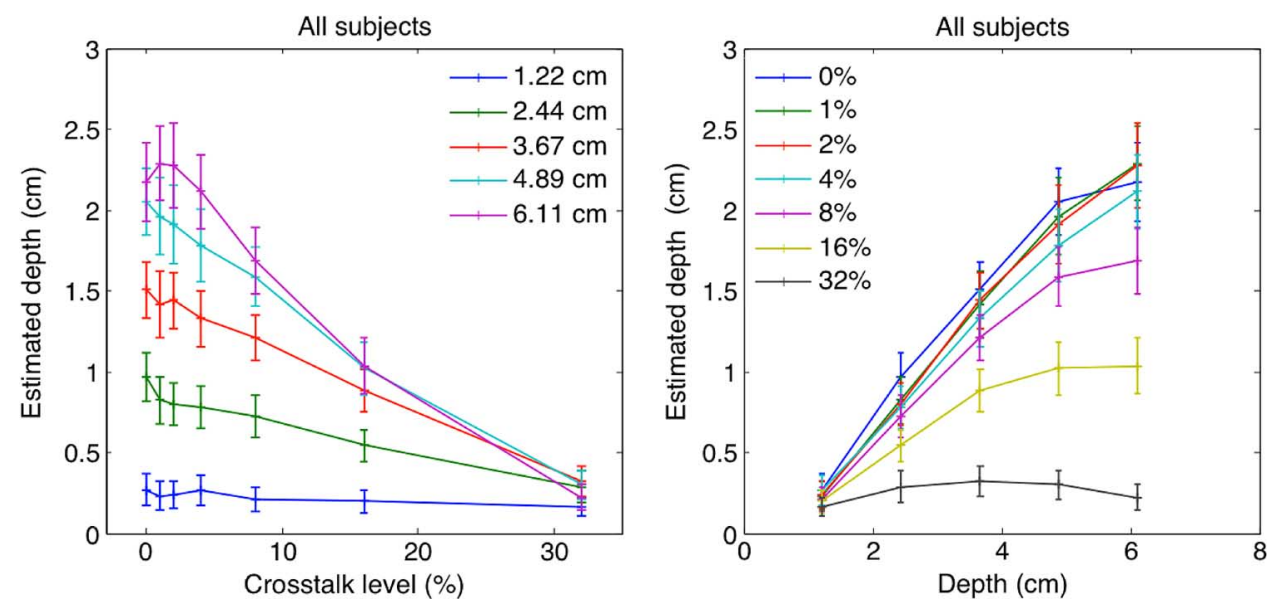

Fig. 3. Results of Experiment 1. The mean data for the nine observers. (Left panel) The abscissa shows the crosstalk levels, and the ordinate shows the depth estimates. (Colored lines) Stimuli with different disparities. The disparities are expressed in terms of the corresponding theoretical depth (see text). (Right panel) The abscissa shows the theoretical depth corresponding to the different disparities, and the ordinate shows the depth estimates. (Colored lines) The stimuli with different crosstalk levels. The error bars indicate $+/-1$ standard error.

discussion and figures to simplify the comparison of perceived depth to theoretical depth. We used a standard formula, which relates disparity to predicted depth at a known viewing distance ([27] pp. 4-5)1. In the computations we used the average interocular distance of our observers $(6.07 \mathrm{~cm})$. The depths relative to the screen corresponding to disparities of $3.54,7.08,10.62$, 14.16 and 17.7 arcmin were $0.61,1.22,1.83,2.44$ and $3.06 \mathrm{~cm}$ accordingly. To determine the total depth between the two lines these values need to be doubled to $1.22,2.44,3.67,4.89$ and $6.11 \mathrm{~cm}$ respectively. In the figures and the discussion we refer to the total depth between the two test lines, not the distance from fixation to one target.

Observers underestimated the depth in the display even in the base condition with $0 \%$ crosstalk. This could have been caused by the observers' underestimation of the viewing distance, which can easily occur in a completely dark room where vergence and accommodation serve as the only cues to distance (for review see [27] section 24.6).

As seen in the left graph of Fig. 3 increasing crosstalk caused a reduction of perceived depth, especially at larger disparities. The effect of crosstalk can be further appreciated in the rightmost graph of Fig. 3 where the mean data were plotted as a function of disparity. If crosstalk had no effect then the lines on this graph would overlap. It is clear that for large disparities depth is reduced at crosstalk levels as low as $4 \%$.

Since there was a large difference between the perceived depth of the largest and the smallest disparities we used, the effect of crosstalk on the smaller disparities might not be appreciable in Fig. 3. Consequently, we normalized the data for each disparity (divided the depth estimates for each disparity by the largest estimate obtained for that disparity) and then combined these data and plotted them as a function of crosstalk in Fig. 4. It can be seen in this figure that depth judgments at all disparities were affected to some degree by crosstalk. The data for smallest disparity showed a decrease at crosstalk levels beyond $4 \%$, however, the variability is quite high for this

\footnotetext{
${ }^{1}$ The formula we used was: $p d=\left(d * D^{2} / I O D\right)$ where $d$ is the relative disparity, $\mathrm{D}$ is the viewing distance and IOD is the inter-occular distance.
}

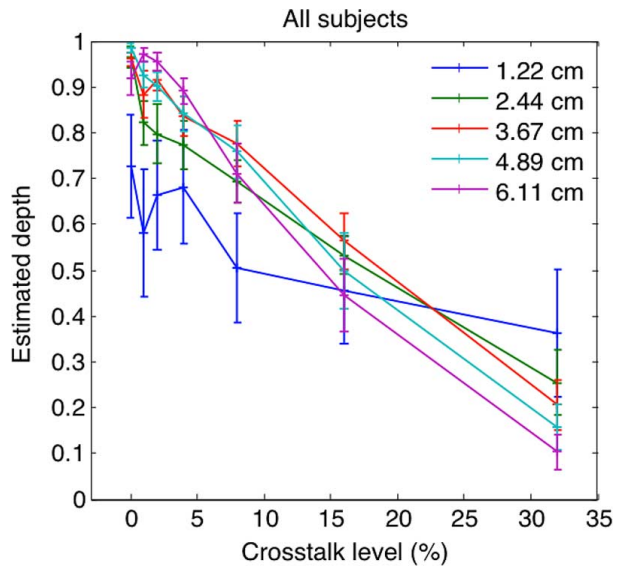

Fig. 4. Results of Experiment 1 with data normalized per each disparity. The abscissa shows the crosstalk levels, and the ordinate shows the normalized depth estimates. (Colored lines) The stimuli with different disparities. The disparities are expressed in terms of the corresponding theoretical depth (see text). The error bars indicate $+/-1$ standard error.

disparity. Generally, larger disparities showed steeper declines with increasing crosstalk and a larger total decrease in perceived depth in comparison to the base level at $0 \%$ crosstalk.

These observations were confirmed by statistical analysis, which is summarized in Table I in Appendix A. For disparities corresponding to depths equal to or larger than $2.44 \mathrm{~cm}$, perceived depth was significantly reduced at 1-8\% (in comparison to the base level at $0 \%$ crosstalk). For the smallest disparity (depth $1.22 \mathrm{~cm}$ ) there was a significant difference between $0 \%$ and $1 \%$ crosstalk, however, the difference is small (only 0.015 $\mathrm{cm})$ and there are no significant differences between the base level with $0 \%$ crosstalk and all the other levels of crosstalk. This indicates that crosstalk might not affect depth perception in this condition. Alternatively, it could mean that for some observers crosstalk did affect depth perception for the smallest disparity and for some there was no effect. Individual data plots from each observer shown on Fig. 2 provide some evidence in support of the latter hypothesis. 


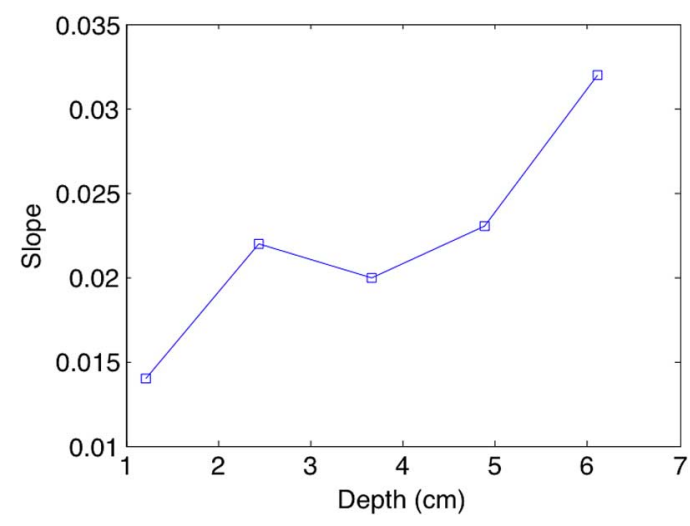

Fig. 5. Mean slopes for the data of Experiment 1. The abscissa shows the different stimulus disparities. The disparities are expressed in terms of the corresponding theoretical depth. The ordinate shows the mean slope. See text for details.

The decline in perceived depth expressed as a percentage tended to increase with increase in disparity (see Table I). For example, the reduction in depth in comparison to the base line at crosstalk $32 \%$ was larger for larger disparities $(41,70,79,85$ and $90 \%$ for depths $1.22,2.44,3.67,4.89$ and $6.11 \mathrm{~cm}$ accordingly). Also see Fig. 9 for comparison of reduction in perceived depth for different disparities.

We also computed the rate of change in perceived depth using the slope of the line between each two consecutive crosstalk levels $(0-1 \%, 1-2 \%, 2-4 \%$ etc.). We have plotted the mean slope for each disparity in Fig. 5. Mean slopes were computed by taking only the slopes corresponding to statistically significant differences between two consecutive crosstalk levels. As can be seen in the figure, the mean slope generally increases with increasing disparity ${ }^{2}$. Taken together the percent decrease in perceived depth and the mean slopes indicate that larger disparities are more affected by crosstalk than the smaller disparities.

\section{Discussion}

Our results confirm previous findings that crosstalk has a detrimental effect on perceived depth from disparity. We showed, with a direct depth estimation task, that the amount of perceived depth decreases in the presence of crosstalk. In general, depth from larger disparities is more affected by crosstalk than depth from smaller disparities. This is not surprising, given that the visibility of the ghost image is correlated with the relative disparity between the left and the right images. The larger the lateral shift of the object in the left eye with respect to the same object in the right eye (i.e. disparity), the larger the distance between the object and the ghost image. Consequently, ghosting increases with increase in disparity [1], [2], [28].

A significant reduction in depth magnitude was observed at crosstalk levels of 1-8\% depending on disparity. For all disparities perceived depth was reduced by about $20 \%$ at crosstalk level of $8 \%$. Beyond $8 \%$ depth was reduced at increasing rates especially for larger disparities. Based on these data we recommend maintaining the crosstalk levels in S3D systems as low as possible but definitely below $8 \%$. The decrease in perceived depth

\footnotetext{
${ }^{2}$ The high slope value for disparity corresponding to depth $2.44 \mathrm{~cm}$ is due to the initial sharp dip in the curve between crosstalk $0 \%$ and $1 \%$ (slope 0.06 ).
}

would likely reduce the quality of S3D images and thus viewer satisfaction.

The effect of crosstalk depends on parameters of the displayed image other than disparity. For example, contrast plays an important role in the perception of ghosting from crosstalk in that larger contrast results in more ghosting [1], [2], [28]. Another important aspect is the nature of the image. Crisp boundaries make ghosting more pronounced, while blurry boundaries disguise it [28]. However, sharp boundaries are also associated with better stereopsis [29]. Color may be a factor in the ghosting phenomenon with brighter colors creating more vivid ghosts than darker colors but, to our knowledge, this aspect has not yet been explored. There is some evidence that thresholds for perceiving ghosting from crosstalk is higher for natural images than for artificial ones [28], [30], however, it is not clear whether there is a similar difference in the effect of crosstalk on the magnitude of depth from disparity.

In our experimental setup we were careful to choose the width of the stimulus lines such that for all the disparities the ghost image would overlap with the original image. This was done to imitate ghosting in large objects for which the ghost image rarely segregates from the original. Natural scenes contain many relatively large objects for which the type of ghosting simulated in our experiment is typical. In experiments reported elsewhere (to be presented at Stereoscopic Displays and Applications 2011) we evaluated the effects of a different type of ghosting, which appears in images containing thin contours (wire fences, tree branches, cords, ropes etc.). In these cases the ghost is separated from the original image even for modest disparity. This type of ghosting might be expected to result in a different percept than the one simulated here due to the possibility of double matching. As in the present study depth degraded with crosstalk but, in contrast to the present study, a significant degradation was found at all disparities.

\section{EXPERIMENT II}

In this experiment we explored the effect of crosstalk on depth magnitude perception from monocular occlusions.

\section{A. Methods}

Observers were the same as in Experiment 1 except that observers AS and AC were replaced with observers DS and SR.

The experimental setup and apparatus were the same as in Experiment 1 but the stimulus differed. The stimulus was composed of a centrally positioned binocular rectangle $(70.8 \times$ 177 arcmin) and a monocular bar $(7.08 \times 132.7 \mathrm{arcmin})$. The bar was placed to the right of the rectangle in the right eye. In this configuration the bar was consistently perceived as occluded by the rectangle and hence positioned behind it in depth (see the discussion of da Vinci stereopsis in the Introduction section). The right edge of the bar was 17.7 arcmin away from the right edge of the rectangle (Fig. 6). Theoretically, at this separation the monocular object should be seen $3.06 \mathrm{~cm}$ away from the occluding rectangle.

The fixation cross, the sliding scale, luminance, crosstalk levels and the statistical analysis were identical to Experiment 1. Since the only difference between the two eyes was the 


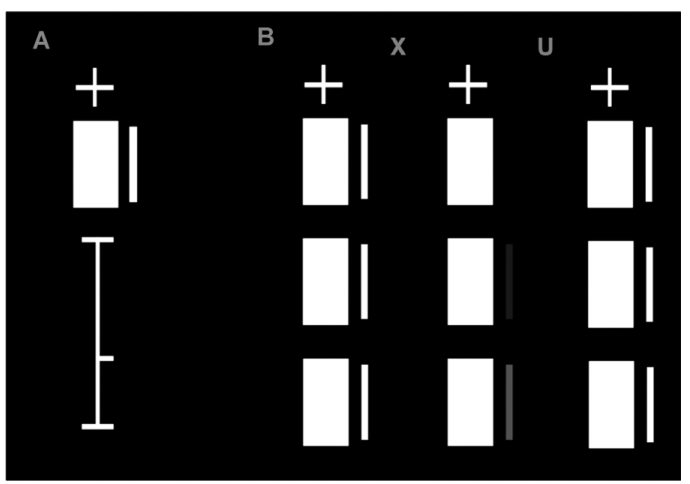

Fig. 6. Graphic depiction of stimuli used in Experiment 2. (a) The complete display. (b) Example of stimuli arranged for (left and middle column) crossed and (middle and right column) divergent fusion. On the top row there is no crosstalk; the middle and bottom rows have $10 \%$ and $32 \%$ crosstalk, respectively.
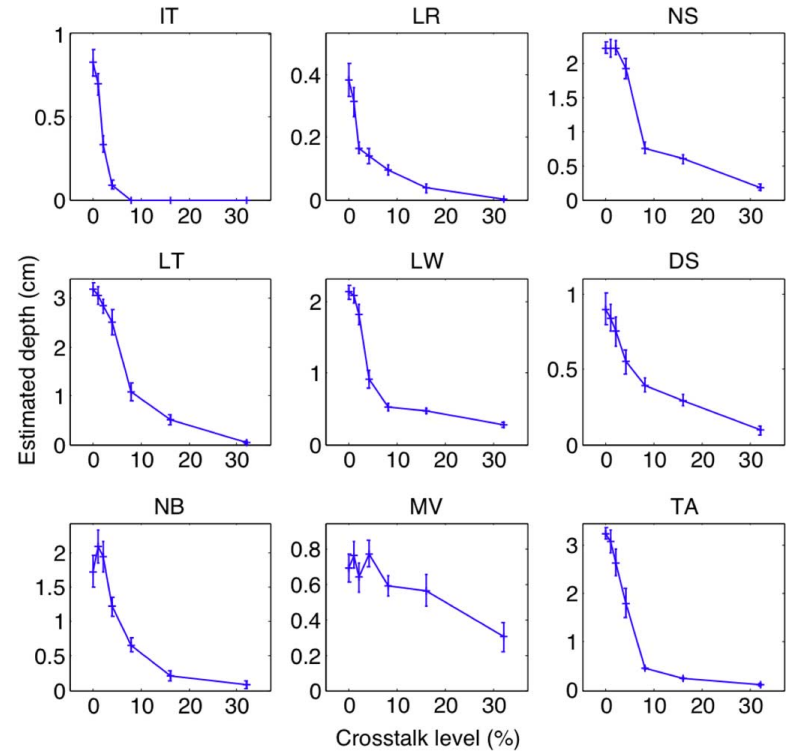

Fig. 7. Results of Experiment 2 for all observers. The abscissa shows the crosstalk levels, and the ordinate shows the normalized depth estimates. The error bars indicate $+/-1$ standard error. Note that the ordinate shows scales tailored to each observer to account for individual differences.

presence of the bar in the right eye, the only perceivable ghost image was that of the bar in the left eye.

The observers were asked to adjust the cursor on the scale to indicate how much depth they perceived between the rectangle and the bar using the procedure described in Experiment 1. The experiment consisted of one session in which each condition (crosstalk level) was presented 20 times in random order (140 trials total per subject).

\section{B. Results}

Original data for all observers are shown on Fig. 7. Mean data for all observers are shown in Fig. 8. The crosstalk levels are plotted on the abscissa and the estimated depth on the ordinate. As in Experiment 1 depth magnitude is underestimated by some observers at $0 \%$ crosstalk (see Fig. 7). The reason for this could be the misestimation of viewing distance as with disparity-based stimuli.

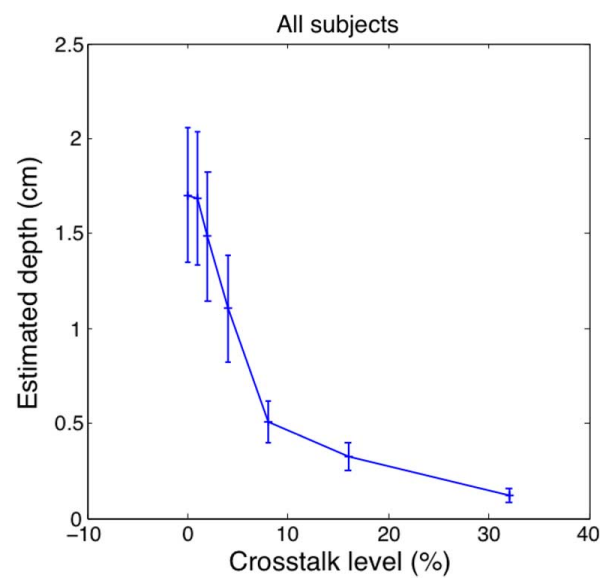

Fig. 8. Mean data of Experiment 2. The abscissa shows the crosstalk levels, and the ordinate shows the normalized depth estimates. The error bars indicate $+/-1$ standard error.

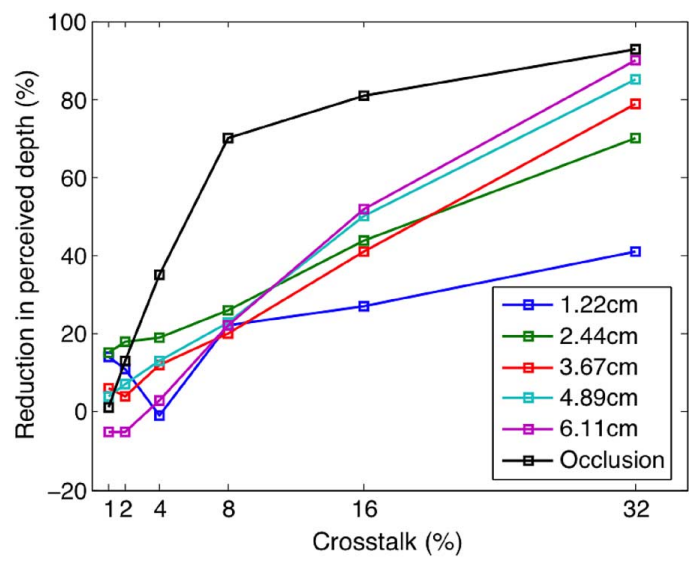

Fig. 9. Comparison of data of Experiments 1 and 2. The abscissa shows the crosstalk levels, and the ordinate shows the reduction of perceived depth (in \%) with respect to the $0 \%$ crosstalk condition. (Colored lines) Disparities tested in Experiment 1 and (black line) the data from Experiment 2.

Fig. 8. shows that crosstalk, even at its lowest levels causes a substantial reduction in perceived depth. Statistical analysis showed significant differences between the base zero-crosstalk condition and all the crosstalk levels larger than $1 \%$ (for exact values see Table II in Appendix A). There was no statistical difference found between the base crosstalk level and crosstalk of $1 \%$. With $2 \%$ crosstalk perceived depth was reduced by $13 \%$, with $4 \%$ percent crosstalk perceived depth was reduced by $35 \%$ and with $8 \%$ crosstalk perceived depth was reduced by $70 \%$.

Interestingly, at high crosstalk levels observers reported perceptual artifacts such as slant and perception of volume instead of a flat bar. These artifacts might further contribute to the degradation of depth perception.

\section{Discussion}

In this experiment we found that crosstalk greatly reduced perceived depth from monocular occlusions. The perceived depth magnitude decreased significantly at crosstalk levels as low as $2 \%$ in comparison to the base level, and was reduced by $70 \%$ at $8 \%$ crosstalk. This effect is greater than the effect of crosstalk on disparity reported in Experiment 1 where the 

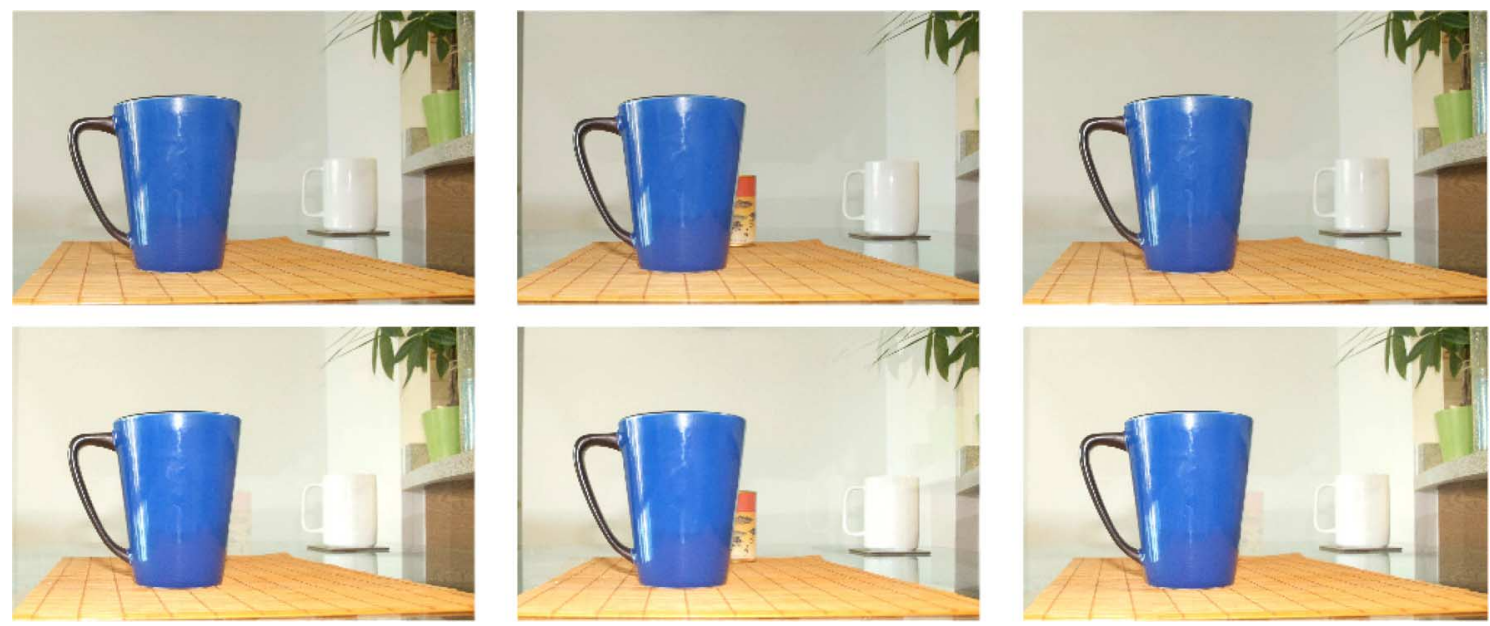

Fig. 10. Illustration of the effect of crosstalk on perceived depth from monocular occlusions in a photograph of a natural scene. The left and center columns are arranged for divergent fusion, and the center and right columns are arranged for crossed fusion. On the top of the panel, there is zero crosstalk, and the tea box can be clearly seen behind the blue cup. On the bottom of the panel, there is $15 \%$ crosstalk, and the depth of the tea box is reduced.

largest reduction in the disparity condition at $8 \%$ crosstalk was $26 \%$. The greater impact of crosstalk on depth from occlusions can be fully appreciated in Fig. 9 where we compared the rate of depth reduction in Experiments 1 and 2. The effect of crosstalk on depth from monocular occlusions is clearly more detrimental than on disparity-defined depth. This difference in effects can be explained by the nature of the occlusion phenomenon. In images without crosstalk, when the occluded object appears in the right eye, there is no corresponding object that can be matched to it in the left eye. It is assumed that the visual system estimates the depth of the monocular object using occlusion geometry [25]. When crosstalk is introduced, the ghost image presents a possible match for the occluded object, albeit, of a different luminance. If the monocular object, such as the bar in our display, is to be matched by the visual system to the ghost image in the other eye, the disparity corresponding to this match will be zero and thus the object should appear at the screen plane. Since the ghost image does not provide a perfect match (due to the difference in luminance) the depth of the monocular occlusion is not reduced completely, but the size of the reduction increases with increasing crosstalk. Moreover, as disparity is considered to be a more reliable cue to depth than monocular occlusion [25], [32], the visual system might prefer the disparity signal provided by the ghost match over the cue provided by the occlusion geometry.

In the case of stimuli containing binocular disparity, the correct match is always present in the image along with the ghost match created by the ghosting. Consequently, the visual system can choose the correct match (with the same luminance) over the ghost match, especially at lower levels of crosstalk. This makes disparity-based depth more robust to ghosting than depth based on monocular occlusion.

The large effect of crosstalk on depth from monocular occlusions is important to consider since monocular occlusions play a significant role in the perception of depth [17] and degraded depth percepts can affect the quality of stereoscopic images. An example of the effect of crosstalk on perceived depth from occlusions in a natural image is shown in Fig. 10.

This is the first report on the perception of depth from monocular occlusions in the context of stereoscopic displays. Monocular occlusions should be studied more closely to understand their contribution to complex images and their effect on depth in S3D media. Correct treatment of monocular occlusions is also critical for synthesis of stereoviews for conversion of 2D content to 3D representation or for multiview displays (see [26]).

\section{CONCLUSION}

We have shown that crosstalk has a detrimental effect on the perceived magnitude of depth from disparity and monocular occlusions. Stimuli in which depth was based on monocular occlusions were more affected by crosstalk than those based on disparity; however, in both types of display perceived depth was significantly reduced at fairly low levels of crosstalk. Our results suggest that for optimal image quality crosstalk levels should be held below $1 \%$. However, most of the depth percept is maintained at crosstalk levels of up to 4\%. At this level of crosstalk in our experiments perceived depth was reduced by $12-19 \%$ for the disparity stimuli and by $35 \%$ for the monocular stimuli. In natural images with lower contrast and other cues to depth the depth reduction effect might not be as pronounced as in our displays.

In 3D television crosstalk can arise due to several reasons. Current 3D TV sets mostly rely on time-sequential stereo with shutter glasses (e.g. 3D ready TVs, Sony, Panasonic) or autostereoscopic technologies (e.g. TCL). These types of 3D displays are prone to crosstalk due to technological imperfections. Moreover, crosstalk can result from compression and transmission distortions. Our work emphasizes the importance of addressing these problems carefully to ensure high quality, vivid depth perception in 3D TV and cinema. 


\section{APPENDIX A}

TABLE I

STATISTICAL ANALYSIS FOR EXPERIMENT I

\begin{tabular}{|c|c|c|c|c|c|}
\hline $\begin{array}{c}\text { Depth } \\
\text { (cm) }\end{array}$ & $\begin{array}{c}\text { Sample 1 } \\
\text { crosstalk } \\
(\%)\end{array}$ & $\begin{array}{c}\text { Sample } 2 \\
\text { crosstalk } \\
(\%)\end{array}$ & p-value & $\begin{array}{c}\text { Diff. in } \\
\text { means } \\
(\mathrm{cm})\end{array}$ & $\begin{array}{c}\text { Diff. in } \\
\text { means } \\
(\%)\end{array}$ \\
\hline \multirow[t]{6}{*}{1.22} & 0 & 1 & $0.029^{*}$ & 0.015 & 14 \\
\hline & 0 & 2 & 0.101 & 0.015 & 11 \\
\hline & 0 & 4 & 0.312 & -0.001 & -1 \\
\hline & 0 & 8 & 0.054 & 0.022 & 22 \\
\hline & 0 & 16 & 0.082 & 0.026 & 27 \\
\hline & 0 & 32 & 0.180 & 0.035 & 41 \\
\hline \multirow[t]{6}{*}{2.44} & 0 & 1 & $0.006^{*}$ & 0.060 & 15 \\
\hline & 0 & 2 & $0.021 *$ & 0.065 & 18 \\
\hline & 0 & 4 & $0.002^{*}$ & 0.077 & 19 \\
\hline & 0 & 8 & $0.002 *$ & 0.105 & 26 \\
\hline & 0 & 16 & $0.002 *$ & 0.175 & 44 \\
\hline & 0 & 32 & $0.002^{*}$ & 0.280 & 70 \\
\hline \multirow[t]{6}{*}{3.67} & 0 & 1 & 0.248 & 0.056 & 6 \\
\hline & 0 & 2 & 0.150 & 0.030 & 4 \\
\hline & 0 & 4 & $0.010^{*}$ & 0.082 & 12 \\
\hline & 0 & 8 & $0.010^{*}$ & 0.127 & 20 \\
\hline & 0 & 16 & $0.002^{*}$ & 0.263 & 41 \\
\hline & 0 & 32 & $0.002 *$ & 0.495 & 79 \\
\hline \multirow[t]{6}{*}{4.89} & 0 & 1 & 0.070 & 0.052 & 4 \\
\hline & 0 & 2 & $0.027^{*}$ & 0.077 & 7 \\
\hline & 0 & 4 & $0.014^{*}$ & 0.128 & 13 \\
\hline & 0 & 8 & $0.002 *$ & 0.203 & 23 \\
\hline & 0 & 16 & $0.002^{*}$ & 0.437 & 50 \\
\hline & 0 & 32 & $0.002 *$ & 0.734 & 85 \\
\hline \multirow[t]{6}{*}{6.11} & 0 & 1 & 0.850 & -0.052 & -5 \\
\hline & 0 & 2 & 0.882 & -0.036 & -5 \\
\hline & 0 & 4 & 0.125 & 0.026 & 3 \\
\hline & 0 & 8 & $0.004 *$ & 0.207 & 22 \\
\hline & 0 & 16 & $0.002^{*}$ & 0.472 & 52 \\
\hline & 0 & 32 & $0.002^{*}$ & 0.815 & 90 \\
\hline
\end{tabular}

TABLE II

STATISTICAL ANALYSIS FOR EXPERIMENT II

\begin{tabular}{|c|c|c|c|c|}
\hline $\begin{array}{c}\text { Sample 1 } \\
\text { crosstalk } \\
(\boldsymbol{\%})\end{array}$ & $\begin{array}{c}\text { Sample 2 } \\
\text { Crosstalk } \\
(\boldsymbol{\%})\end{array}$ & p-value & $\begin{array}{c}\text { Diff. in } \\
\text { means } \\
(\mathbf{c m})\end{array}$ & $\begin{array}{c}\text { Diff. in } \\
\text { means } \\
(\boldsymbol{\%})\end{array}$ \\
\hline 0 & 1 & 0.180 & 0.018 & 1 \\
\hline 0 & 2 & $0.021^{*}$ & 0.218 & 13 \\
\hline 0 & 4 & $0.004^{*}$ & 0.599 & 35 \\
\hline 0 & 8 & $0.002 *$ & 1.196 & 70 \\
\hline 0 & 16 & $0.002^{*}$ & 1.378 & 81 \\
\hline 0 & 32 & $0.002^{*}$ & 1.582 & 93 \\
\hline
\end{tabular}

\section{ACKNOWLEDGMENT}

The authors would like to thank Lindsay Rubinfeld and Tetyana Andriychuk for helping with data collection. The support of the Ontario Centres of Excellence and the Ontario Media Development Corporation to the 3D Film Consortium (3DFLIC) is greatly appreciated.

\section{REFERENCES}

[1] P. J. H. Seuntiens, L. M. J. Meesters, and W. A. IJsselsteijn, "Perceptual attributes of crosstalk in 3D images," Displays, vol. 26, pp. 177-183, 2005.

[2] F. Kooi and A. Toet, "Visual confort of binocular and 3D displays," Displays, vol. 25, pp. 99-108, 2004.
[3] L. M. WIlcox and J. A. D. Stewart, "Determinants of perceived image quality: Ghosting vs. brightness," in Stereoscopic Displays and Virtual Reality Syst. X, 2003.

[4] A. J. Woods, "Understanding crosstalk in stereoscopic displays," in Three-Dimensional Syst, Appl., 2010.

[5] A. J. Woods and S. S. L. Tan, "Characterising sources of ghosting in time-sequential stereoscopic video displays," in Stereoscopic Displays and Virtual Reality Syst. IX, 2002.

[6] T. Jarvenpaa and M. Salmimaa, "Optical characterization of autostereoscopic 3-D displays," .J. Soc. Inf. Display, vol. 16, pp. 825-833, 2008.

[7] M. Salmimaa and T. Jarvenpaa, "3-D crosstalk and luminance uniformity from angular luminance profiles of multiview autostereoscopic 3-D displays," Journal of Society for Information Display, vol. 16, pp. 1033-1040, 2008.

[8] A. J. Woods and C. R. Harris, "Comparing levels of crosstalk with red/ cyan, blue/yellow, and green/magenta anaglyph 3D glasses," in Stereoscopic displays and applications XXI, 2010, pp. 0Q1-0Q12.

[9] H. Jorke and M. Fritz, Stereo projection using interference filters, $\mathrm{p}$. 60550G, 2006

[10] A. J. Woods and T. Rourke, "Ghosting in anaglyphic stereoscopic images," in Stereoscopic Displays and Virtual Reality Syst. XI, 2004.

[11] C. C. Pan, Y. R. Lee, K. F. Huang, and T. C. Huang, "Crosstalk evaluation of shutter-type stereoscopic 3D display," in Soci. for Inf. Display, 10 2010, pp. 128-131.

[12] S. Pala, R. Stevens, and R. Surman, "Optical crosstalk and visual comfort of a stereoscopic display used in a real-time application," in Stereoscopic Displays and Virtual Reality Syst. XIV, 2007, pp. 649011.1-649011.12.

[13] M. Lambooij, M. F. Fortuin, W. A. IJsselsteijn, and I. Heynderickx, "Visual discomfort associated with 3D displays," in 5th Int. Workshop Video Process. Quality Metrics for Consum. Electron., 2010.

[14] M. Lambooij and W. A. IJsselsteijn, "Visual discomfort and visual fatigue of stereoscopic displays: a review," J. Imaging Sci. Technol., vol. 53, pp. 030201-1-030201-14, 2009.

[15] K. C. Huang, J. C. Yuan, W. J. Hsueh, and N. Y. Wang, "A study of how crosstalk affects stereopsis in stereoscopic displays," in Stereoscopic Displays and Virtual Reality Syst. X, 2003, pp. 247-253.

[16] K. Nakayama and S. Shimojo, "Da Vinci stereopsis: Depth and subjective occluding contours from unpaired image points," Vision Res., vol. 30, pp. 1811-1825, 1990.

[17] J. M. Harris and L. M. Wilcox, "The role of monocularly visible regions in depth and surface perception," Vision Res., vol. 49, pp. 2666-2685, 2009.

[18] B. Gillam and E. Borsting, "The role of monocular regions in stereoscopic displays," Perception, vol. 17, pp. 603-608, 1988.

[19] P. M. Grove and H. Ono, "Ecologically invalid monocular texture leads to longer perceptual latencies in random-dot stereograms," Perception, vol. 28, pp. 627-639, 1999.

[20] L. M. Wilcox and D. C. Lakra, "Depth from binocular half-occlusions in stereoscopic images of natural scenes," Perception, vol. 36, pp. 830-839, 2007.

[21] B. L. Anderson and K. Nakayama, "Toward a general theory of stereopsis: Binocular matching, occluding contours, and fusion," $\mathrm{PSYCHO}$ LOGICAL REVIEW, vol. 101, pp. 414-445, 1994.

[22] J. Hakkinen and G. Nyman, "Phantom surface captures stereopsis," Vision Res., vol. 41, pp. 187-199, 2001.

[23] B. Gillam and K. Nakayama, "Quantitative depth for a phantom surface can be based on cyclopean occlusion cues alone," Vision Res., vol. 39, pp. 109-112, 1999.

[24] L. Liu, S. B. Stevenson, and C. M. Schor, "Quantitative stereoscopic depth without binocular correspondence," Nature, vol. 367, pp. 66-68, 1994.

[25] I. Tsirlin, L. M. Wilcox, and R. S. Allison, "Monocular occlusions determine the perceived shape and depth of occluding surfaces," J. Vision, vol. 10, 2010.

[26] L. M. Wilcox, I. Tsirlin, and R. S. Allison, "Sensitivity to monocular occlusions in stereoscopic imagery: Implications for S3D content creation, distribution and exhibition," in SMPTE Int. Conf. Stereoscopic 3 D Media \& Entertainment, 2010

[27] I. P. Howard, "Seeing in Depth," I. Porteous, pp. 271-317, 2002.

[28] A. Hanazato, M. Okui, H. Yamanoue, and I. Yuyama, "Evaluation of crosstalk in stereoscopic display," in 3D Image Conf., 1999, pp. 258-263.

[29] C. M. Schor and I. Wood, "Disparity range for local as a function of luminance spatial frequency," Vision Res., vol. 23, pp. 1649-1654, 1983.

[30] L. Lipton, "Factors affecting ghosting in time-multiplexed planostereoscopic CRT display systems," in SPIE, 1987. 
[31] J. D. Krol and W. A. v. d. Grind, "The double-nail illusion: Experiments on binocular vision with nails, needles and pins," Perception, vol. 9, pp. 651-669, 1980.

[32] J. Hakkinen and G. Nyman, "Depth assymetry in daVinci stereopsis," Vision Res., vol. 36, pp. 3815-3819, 1996.

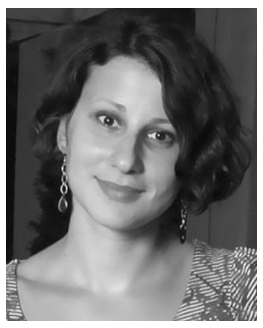

Inna Tsirlin received the B.Sc. (Spec. Hons.) and the M.Sc. in computer science from York University. She working toward the Ph.D. degree in psychology, working with Dr. Wilcox and Dr. Allison at the Centre for Vision Research at York University in Toronto, Canada, and is also completing the Neuroscience Diploma Program at York.

Her doctoral research involves studying various aspects of depth perception from binocular disparity and monocular occlusions using a combination of psychophysical and computational methods. In particular, she is interested in the interaction between disparity and occlusion cues, S3D cinematography, stereoscopic transparency and disparity interpolation.

She is a recipient of the NSERC Alexander Graham Bell Canada Graduate Scholarship. More information about Inna's research can be found on her website: http://www.wilcoxlab.yorku.ca/ inna.

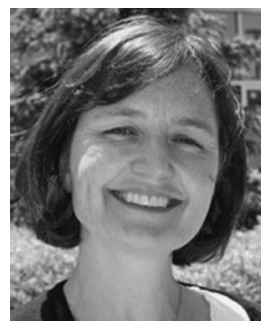

Laurie Wilcox received the M.A. and Ph.D. degrees from the University of Western Ontario, Canada.

She is an Associate Professor of Psychology, and Associate Director of the Centre for Vision Research at York University, Toronto. She is also cross-appointed to the graduate program in Biology at York University. She joined the Vision Group in the Department of Ophthalmology at McGill University. In 1995, she became a Chercheur Boursier at the University of Montreal. In 1996, she was offered a tenure-stream faculty position at York University. During this time she developed a strong research program focused on fundamental properties of human stereoscopic depth perception. Her research uses psychophysical techniques to reveal properties of the neural mechanisms, which underpin stereoscopic (S3D) depth perception. In addition to basic research on S3D she has been actively involved in understanding the factors that influence viewer comfort and satisfaction when watching large format S3D film. To this end she has collaborated with industry partners such as IMAX to examine issues such as the effects of vertical misalignment of S3D imagery on audience satisfaction. Most recently she has also engaged with the S3D film community, giving workshops and lectures on the fundamentals of stereopsis and participating in panel discussions at film festivals.

Dr. Wilcox received a prestigious five-year NSERC Women's Faculty Award in 1995. Her research has been supported a new investigator CFI award, and grants from NSERC, the Ontario Centres of Excellence, and most recently through a New Media Initiative (partnership between NSERC and Canada Council of the Arts).

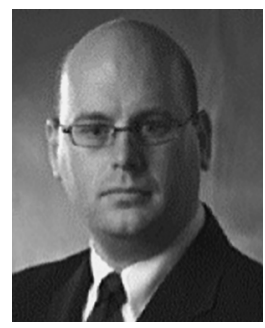

Robert Allison received the B.Sc. in computer engineering from the University of Waterloo in 1991,the M.Sc. in electrical engineering (biomedical engineering) from the University of Toronto, Canada, and the Ph.D. specializing in stereoscopic vision from York University, Toronto, Canada, in 1998.

$\mathrm{He}$ is Associate Professor of Computer Science and Engineering at York University, joining the faculty in 2001 . He is also appointed to the graduate program in Psychology at York. After graduating from York University, he worked as an Electrical Engineer at Atlantis Aerospace in Brampton, Canada, where he designed electronics for flight training devices. He was on the experimental team for the 1998 Neurolab space shuttle mission and did post-doctoral research at York University and the University of Oxford. He works on perception of space and self-motion in virtual environments, the measurement and analysis of eye-movements and stereoscopic vision. His research uses psychophysical and computational techniques to study how we can reconstruct and navigate the three-dimensional world around us from the two-dimensional images on the retinas. His research enables effective technology for advanced virtual reality and augmented reality and for the design of stereoscopic displays.

Dr. Allison is recipient of the Premier's Research Excellence Award from the Province of Ontario in recognition for research in human stereoscopic vision and depth perception. He has also received a McDonnell-Pew Visiting Fellowship, an NSERC Postdoctoral Fellowship and an Australian Research Council International Fellowship. He is a Senior Member of the Institute of Electrical and Electronic Engineers and also a member of the IEEE Computer Society and the Association for Computing Machinery. 